\title{
Network Performance of Asynchronous UTRA-like FDD/CDMA Systems using Loosely Synchronised Spreading Codes
}

\author{
Song Ni, Hua Wei, Jonathan S. Blogh, Lajos Hanzo ${ }^{1}$ \\ Dept. of Electronics and Computer Science, Univ. of Southampton, SO17 1BJ, UK. \\ Tel: +44-(0)23-8059-3125, Fax: +44-(0)23-8059-4508 \\ Email: 1h1@ecs.soton.ac.uk http://www-mobile.ecs.soton.ac.uk
}

\begin{abstract}
In this paper we characterize the capacity of a UTRA-like Frquency Division Duplex (FDD) Code Division Multiple Access (CDMA) system employing Loosely Synchronized (LS) spreading codes. Current CDMA systems are interference limited, suffering from Inter-Symbol-Interference (ISI), since the orthogonality of the spreading sequences is destroyed by the channel. They also suffer from MultipleAccess-Interference (MAI) owing to the non-zero cross- correlations of the spreading codes. LS codes exhibit a socalled Interference Free Window (IFW), where both the auto-correlation and cross-correlation of the codes become zero. Therefore LS codes have the promise of mitigating the effects of both ISI and MAI in time dispersive channels. Hence, LS codes have the potential of increasing the capacity of CDMA networks. This contribution studies the achievable network performance by simulation and compares it to that of a UTRA-like FDD/CDMA system using Orthogonal Variable Rate Spreading Factor (OVSF) codes.
\end{abstract}

\section{INTRODUCTION}

In our previous research $[1,2,3]$, the performance of a UTRAlike FDD CDMA system was quantified, when supported by adaptive beam-steering and adaptive modulation [4]. In [3], the system employed OVSF spreading codes [5], which offer the benefit of perfect orthogonality in an ideal channel. In a non-dispersive channel, all intracell users' signals are perfectly orthogonal. However, upon propagating through a dispersive multipath channel this orthogonality is eroded, hence all other users will interfere with the desired signal. Therefore in practice the intra-cell interference is always non-zero.

In this contribution, we will consider the employment of a specific family of spreading codes, which are known as Loosely Synchronized (LS) codes [6]. These codes exhibit a so-called Interference Free Window (IFW), where the off-peak aperiodic autocorrelation values as well as the aperiodic cross-correlation values become zero, resulting in zero ISI and zero MAI, provided that the delayed asynchronous transmissions arrive within

The financial support of the European Union under the auspices of the SCOUT project is gratefully acknowledged. the IFW. More specifically, interference-free CDMA communications become possible, when the total time offset expressed in terms of the number of chip intervals, which is the sum of the time-offset of the mobiles plus the maximum channel-induced delay spread is within the code's IFW [7]. By employing this specific family of codes, we are capable of reducing the ISI and MAI, since users in the same cell do not interference with each other, as a benefit of the IFW provided by the LS codes used.

The outline of this paper is as follows. Firstly, we briefly focus our attention on the design of LS codes in the next section. Section 3 introduces the system parameters, leading to a discussion on the performance metrics employed in Section 4. The system performance benefits of using LS codes in a pedestrian scenario are quantified in Section 5 and we conclude our discussions in Section 6 .

\section{LOOSELY SYNCHRONIZED CODES}

There exists a specific family of LS codes [6], which exhibits an IFW. Specifically, LS codes exploit the properties of the so-called orthogonal complementary sets $[6,8]$. To expound further, let us introduce the notation of $\operatorname{LS}\left(N, P, W_{0}\right)$ for denoting the family of LS codes generated by applying a $(P \times P)$ dimensional Walsh-Hadamard matrix to an orthogonal complementary code set of length $N$, while inserting $W_{0}$ number of zeros in the centre and at the beginning of the LS code, as shown in Figure 1, using the procedure described in [6]. Then, the total length of the $\operatorname{LS}\left(N, P, W_{0}\right)$ code is given by $L=2 N P+2 W_{0}$ and the number of codes available is given by $2 P$.

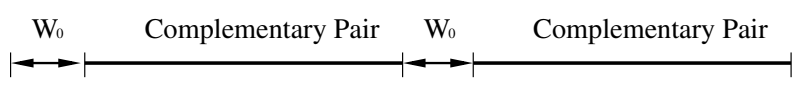

Figure 1: The LS code structure.

Since the construction method of binary LS codes was described in [6], we will focus our attention on the employment of orthogonal complementary sets $[9,10]$ for the generation of LS codes. Firstly, we define a sequence set $\mathbf{c}_{1}, \ldots, \mathbf{c}_{N}$, where $\mathbf{c}_{n}=\left[c_{n, 0}, \ldots, c_{n, L-1}\right]$ is a spreading sequence having a length of $L$. These spreading codes exhibit an IFW width of $\tau_{\mathrm{IFW}}$, if the crosscorrelation of the spreading codes satisfies: 
$R_{j k}(\tau)=\sum_{l=0}^{L-1} c_{j, l} c_{k,(l+\tau) \bmod L}= \begin{cases}L, & \text { for } \tau=0, j=k \\ 0, & \text { for } \tau=0, j \neq k \\ 0, & \text { for } 0<|\tau| \leq \tau_{\mathrm{IFW}}\end{cases}$

The aperiodic correlation $R_{j, k}(\tau)$ of two sequences $\mathbf{g}_{j}$ and $\mathbf{g}_{k}$ has to satisfy Equation 1 for the sake of maintaining an IFW of $\tau_{\mathrm{IFW}}$ chip intervals.

For a given complementary pair $\left\{\mathbf{c}_{0}, \mathbf{s}_{0}\right\}$ of length $N$, one of the corresponding mate pairs can be written as $\left\{\mathbf{c}_{1}, \mathbf{s}_{1}\right\}$, where we have:

$$
\begin{aligned}
& \mathbf{c}_{1}=\tilde{\mathbf{s}}_{0}^{*}, \\
& \mathbf{s}_{1}=-\tilde{\mathbf{c}}_{0}^{*},
\end{aligned}
$$

and where $\tilde{\mathbf{s}}_{0}$ denotes the reverse-ordered sequence, while $-\mathbf{s}_{0}$ is the negated version of $\mathbf{s}_{\mathbf{0}}$, respectively. Note that in (2) and (3) additional complex conjugation of the polyphase complementary sequences is required for deriving the corresponding mate pair in comparison to binary complementary sequences [6]. Having obtained a complementary pair and its corresponding mate pair, we may employ the construction method of [6] for generating a family of LS codes. The LS codes generated exhibit an IFW, where we have $R_{j k}(\tau)=0$ for $|\tau| \leq \min \{N-$ $\left.1, W_{0}\right\}$. Hence, we may adopt a choice of $W_{0}=N-1$ in order to minimize the total length of the LS codes generated, while providing as long an IFW as possible.

For example, the LS $(4,2,3)$ codes can be generated based on the complementary pair of [9]:

$$
\begin{aligned}
& \mathbf{c}_{0}=+++- \\
& \mathbf{s}_{0}=++--
\end{aligned}
$$

Upon substituting (2) and (3) into (4) and (5), the corresponding mate pair can be obtained as:

$$
\begin{aligned}
& \mathbf{c}_{1}=\tilde{\mathbf{s}}_{0}^{*}=+-++ \\
& \mathbf{s}_{1}=-\tilde{\mathbf{c}}_{0}^{*}=+---.
\end{aligned}
$$

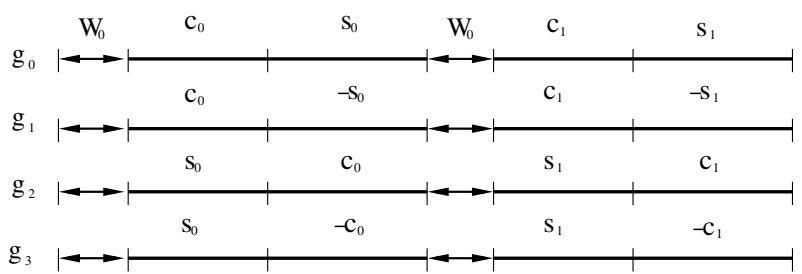

Figure 2: Generating four LS codes

The generation of this set of the four LS codes can be viewed in Fig 2 . Upon invoking the $2 \times 2$-dimensional Hadamard expansion of [6] in the context of the above orthogonal complementary pairs, we can generate a family of four $\operatorname{LS}(4,2,3)$ codes, which are denoted by $\mathbf{g}_{p}, p=0, \cdots, 3$.

All four different codes of the LS $(4,2,3)$ code family exhibited the same autocorrelation magnitudes, namely that seen in Figure 3(a). It can be observed in Figure 3(a) that the offpeak autocorrelation $R_{p}[\tau]$ becomes zero for $|\tau| \leq W_{0}=3$. The

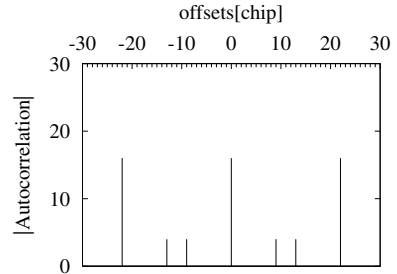

(a) Autocorrelation

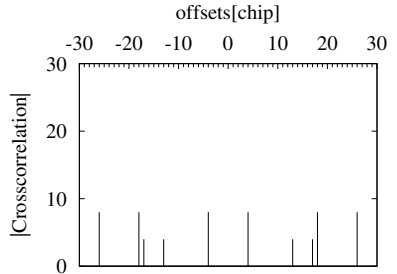

(b) Crosscorrelations
Figure 3: correlation magnitudes of the $\operatorname{LS}(4,2,3)$ codes. (a) All four codes exhibit the same autocorrelation magnitude. (b) The crosscorrelation magnitudes of $\mathbf{g}_{0}$ and $\mathbf{g}_{2}$.

crosscorrelation magnitudes $\left|R_{j, k}(\tau)\right|$ depicted in Figure 3(b) are also zero for $|\tau| \leq W_{0}=3$. Based on the observations made as regards to the aperiodic correlations we may conclude that the $\mathrm{LS}(4,2,3)$ codes exhibit an IFW of \pm 3 chip durations.

\section{SYSTEM PARAMETERS}

New call channel allocation requests were placed in a resource allocation queue for up to $5 \mathrm{~s}$. If during this period a call was not serviced, it was classed as blocked. The mobiles moved freely, in random directions, at a speed of $30 \mathrm{mph}$ within the simulation area, which consisted of 49 cells. The cell-radius was $86.8 \mathrm{~m}$, which was the maximum affordable cell radius for the IFW duration of \pm 3 chip intervals at a chip rate of 3.84 Mchip/s. The call duration and inter-call periods were Poisson distributed with the mean values shown in Table 1. For our initial investigations we have assumed that the basestations and mobiles form a synchronous network.

Furthermore, the post-despreading SINRs required for obtaining the target BERs were determined with the aid of physicallayer simulations using a 4-QAM modulation scheme, in conjunction with 1/2-rate turbo coding for transmission over a COST 207 seven-path Bad Urban channel [11]. Using this turbo-coded transceiver and LS codes having a spreading factor (SF) of 16, the post-despreading SINR required for maintaining the target BER of $1 \times 10^{-3}$ was $6.2 \mathrm{~dB}$. The BER which was deemed to correspond to low-quality access, was stipulated at $5 \times 10^{-3}$. This BER was exceeded for SINRs falling below $5.2 \mathrm{~dB}$. Furthermore, a low-quality outage was declared, when the BER of $1 \times 10^{-2}$ was exceeded, which was encountered for SINRs below $4.8 \mathrm{~dB}$. These values can be seen along with the other system parameters in Table 1.

\section{PERFORMANCE METRICS}

There are several performance metrics that can be used for quantifying the performance or quality of service provided by a mobile cellular network. The following performance metrics have been widely used in the literature and were also advocated by Chuang [13]:

- New call blocking probability, $P_{B}$.

- Call dropping or forced termination probability, $P_{F T}$. A call is dropped when the lower of the uplink and down- 


\begin{tabular}{|l|r||l|r|}
\hline Parameter & Value & Parameter & Value \\
\hline \hline Noisefloor & $-100 \mathrm{dBm}$ & Pilot power & $-8.5 \mathrm{dBm}$ \\
Frame length & $10 \mathrm{~ms}$ & Cell radius & $86.8 \mathrm{~m}$ \\
Multiple access & FDD/CDMA & Number of basestations & 49 \\
Modulation scheme & 4QAM/QPSK & Spreading factor & 16 \\
Min BS transmit power & $-47.5 \mathrm{dBm}$ & Min MS transmit power & $-47.5 \mathrm{dBm}$ \\
Max BS transmit power & $17.5 \mathrm{dBm}$ & Max MS transmit power & $17.5 \mathrm{dBm}$ \\
Power control stepsize & $1 \mathrm{~dB}$ & Power control hysteresis & $1 \mathrm{~dB}$ \\
Low quality access SINR & $5.2 \mathrm{~dB}$ & Outage (1\% BER) SINR & $4.8 \mathrm{~dB}$ \\
Pathloss exponent & -2.0 & Size of Active BS Set (ABS) & 2 \\
Average inter-call-time & $300 \mathrm{~s}$ & Max. new-call queue-time & $5 \mathrm{~s}$ \\
Average call length & $60 \mathrm{~s}$ & Pedestrian speed & $3 \mathrm{mph}$ \\
Max consecutive outages & 5 & Signal bandwidth & $5 \mathrm{MHz}$ \\
Target SINR & $6.2 \mathrm{~dB}$ & & \\
\hline
\end{tabular}

Table 1: Simulation parameters.

link SINRs dips consecutively below the outage SINR (1\% BER) a given number of times.

- Probability of a low quality access, $P_{l o w}$, quantifies the chances of either the uplink or downlink signal quality being sufficiently poor, resulting in a low quality access (0.5\% BER).

- Probability of outage, $P_{\text {out }}$, is defined as the probability that the SINR is below the value at which the call is deemed to be in outage, namely below $4.8 \mathrm{~dB}$, as seen in Table 1.

- Grade-Of-Service (GOS) was defined by Cheng and Chuang [13] as :

Our network performance studies were conducted with aim of maintaining: $P_{B} \leq 3 \%, P_{F T} \leq 1 \%, P_{l o w} \leq 1 \%$ and GOS $\leq 4 \%$.

\section{SIMULATION RESULTS}

Our current research is building on our previous findings recorded in the context of a UTRA-like FDD system [1], where we found that invoking adaptive modulation as well as beam-steering proved to be a powerful means of enhancing the capacity of FDD/CDMA. In the investigations of [1], OVSF codes were used as spreading codes. However, the intracell interference is only eliminated by employing orthorgonal OVSF codes, if the system is perfectly synchronous and provided that the mobile channel does not destroy the OVSF codes' orthogonality. In an effort to prevent intracell interference, again, in this paper we employ LS codes, which exhibit ideal auto-correlation and cross-correlation functions within the IFW. Thereby, the "near far effect" may be significantly reduced and hence the user capacity of the system can be substantially enhanced. Figure 4 compares the BER performance of OVSF codes and LS codes, which were determined with the aid of physical-layer simulations using a 4-QAM modulation scheme, 1/2-rate turbo coding and a Minimum Mean Squared Error Block Decision Feedback Equaliser (MMSE-BDFE) based Multi-User Detector (MUD) [12] joint detection for transmission over a COST
207 seven-path Bad Urban channel [11]. The figure illustrates that the achievable BER performance of LS codes is better than that of OVSF codes. For a spreading factor of 16, the postdespreading SINR required for maintaining a BER of $1 \times 10^{-3}$ was $6.2 \mathrm{~dB}$ in case of LS codes, which is almost $2 \mathrm{~dB}$ lower than that necessitated by the OVSF codes.

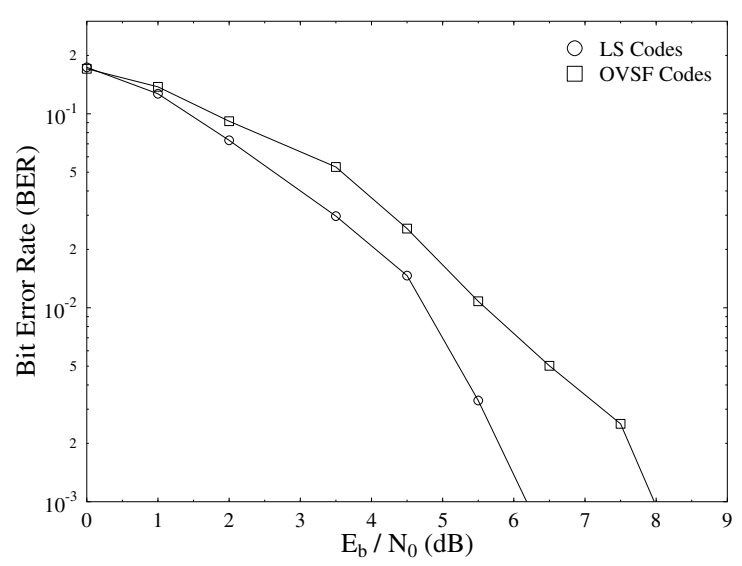

Figure 4: BER performance of a UTRA-like system using OVSF codes and LS codes generated with the aid of physicallayer simulations using 4-QAM modulation, 1/2-rate turbo coding and MMSE-BDFE joint detection for transmissions over a COST 207 seven-path Bad Urban channel.

Figure 5 shows the call dropping probability associated with a variety of traffic loads measured in terms of the mean normalized carried traffic expressed in Erlangs $/ \mathrm{km}^{2} / \mathrm{MHz}$ when subjected to $0.5 \mathrm{~Hz}$ frequency shadowing having a standard deviation of $3 \mathrm{~dB}$. The figure illustrates that the network's performance was significantly improved by using LS codes. In conjunction with OVSF codes, the "No beamforming" scenario suffered from the highest call dropping probability of the six traffic scenarios at a given load. Specifically, the network capacity was limited to 152 users, or to a teletraffic load of approximately 2.65 Erlangs $/ \mathrm{km}^{2} / \mathrm{MHz}$. With the advent of em- 


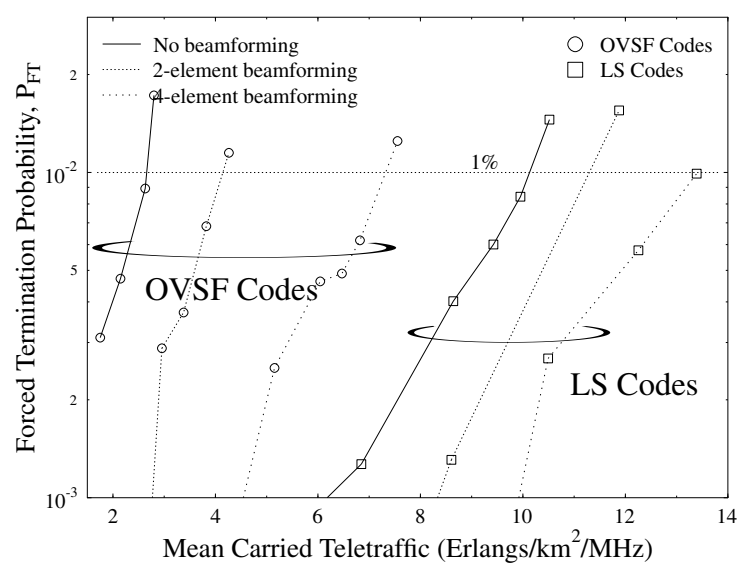

Figure 5: Call dropping probability versus mean carried traffic of the UTRA-like FDD cellular network using LS codes and OVSF codes both with as well as without beamforming in conjunction with shadowing having a frequency of $0.5 \mathrm{~Hz}$ and a standard deviation of $3 \mathrm{~dB}$ for a spreading factor of $\mathrm{SF}=16$.

ploying four-element adaptive antenna arrays at the base stations the number of users supported by the network increased to 428 users, or almost to 7.23 Erlangs $/ \mathrm{km}^{2} / \mathrm{MHz}$. However, in conjunction with LS codes, and even without employing antenna arrays at the base stations the network capacity was dramatically increased to 581 users, or 10.10 Erlangs $/ \mathrm{km}^{2} / \mathrm{MHz}$. When four-element adaptive antenna arrays was employed in LS codes scenario, the system could support 800 users, and equal to a teletraffic load of $13.3916 \mathrm{Erlang} / \mathrm{km}^{2} / \mathrm{MHz}$. This is because the LS codes' perfect auto-correlation and crosscorrelation functions essentially eliminated the intracell interference, as it was discussed in Sections 1 and 2.

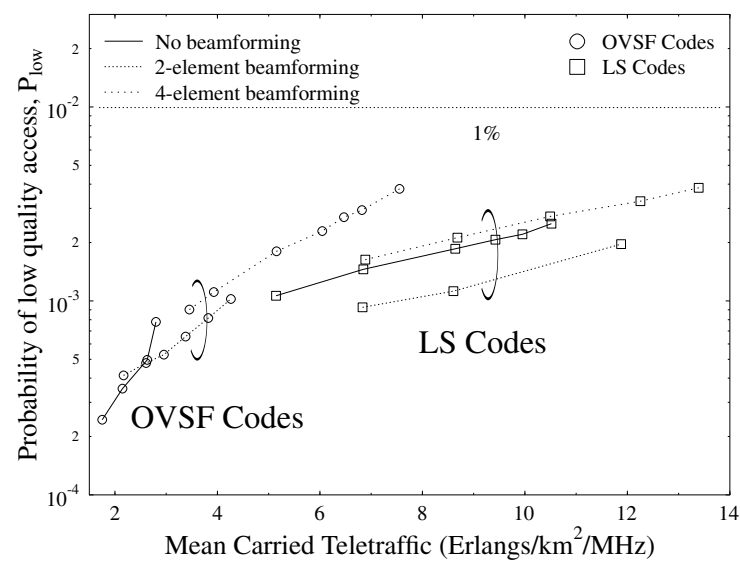

Figure 6: Probability of low quality access versus number of users of the UTRA-like FDD cellular network using LS codes and OVSF codes both with as well as without beamforming in conjunction with shadowing having a frequency of 0.5 and a standard deviation of $3 \mathrm{~dB}$ for a spreading factor of $\mathrm{SF}=16$.
The probability of low quality access is depicted in Figure 6. As expected, a given $P_{l o w}$ value was associated with a higher traffic load, when the number of antenna elements was increased. In the case of OVSF codes, it can be seen from the figure that without beamforming the system suffered more interference as the traffic loads increased, the probability of low quality access became higher. In conjunction with beamforming, intra- and inter-interference was reduced sufficiently, the probability of low quality reduced as well. However, increasing the number of antenna elements from two to four results in an increased probability of a low quality outage owing to the sharper antenna directivity. As a benefit of employing LS codes, the intra-interference was eliminated efficiently, the probability of low quality access was found to be lower even without beamforming, than that of the system using OVSF codes and employing 2- or 4-element beamforming. Again, owing to the sharper antenna directivity, the probability of a low quality outage increased while increasing the number of antenna elements from two to four. It should be noted that the probability of low quality access always remained below our $1 \%$ constraint in the scenarios studied.

Figure 7 shows the achievable Grade-Of Service (GOS) for a range of teletraffic loads. Similar trends were observed regarding the probability of low quality access to those shown in Figure 6. The grade of service is better (i.e. lower) when the traffic load is low, and vice versa for high traffic loads. This is mainly attributable to the higher call blocking probability of the "No beamforming" scenario, particularly in the region of the highest traffic loads.

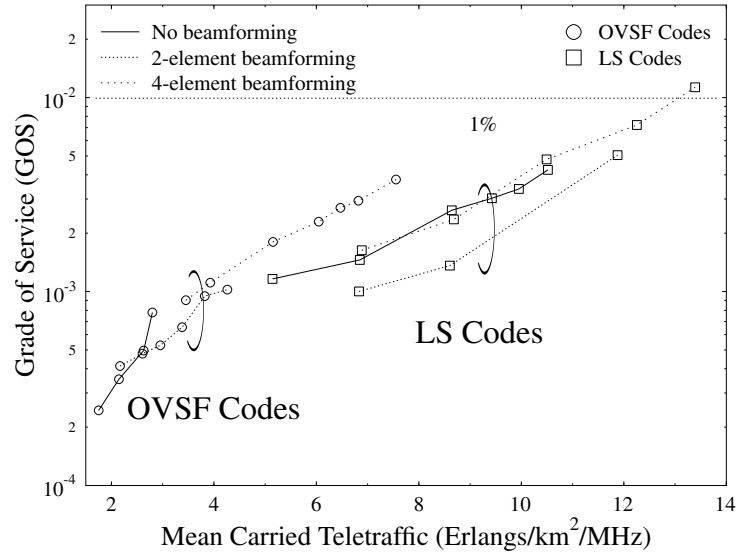

Figure 7: Grade-Of-Service (GOS) versus number of users of the UTRA-like FDD cellular network using LS codes and OVSF codes both with as well as without beamforming in conjunction with shadowing having a frequency of $0.5 \mathrm{~Hz}$ and a standard deviation of $3 \mathrm{~dB}$ for a spreading factor of $\mathrm{SF}=16$.

The mean transmission power versus teletraffic performance is depicted in Figure 8. Again, as a benefit of employing LS codes, both the required mean uplink and downlink transmission power are lower than that necessitated by OVSF codes. The employment of adaptive antenna arrays can result in the attenuation of the desired signal, this is performed in order 


\begin{tabular}{|l|l||l|c|l|l|}
\hline & \multirow{2}{*}{ Spreading Code } & Beamforming & Users & Traffic (Erlangs & \multicolumn{2}{c|}{ Power $(\mathrm{dBm} / \mathrm{MHz})$} & MS & $\mathrm{BS}$ \\
\hline \hline OVSF codes & No & 152 & 2.65 & -9.0 & -9.0 \\
\hline OVSF codes & 2-elements & 242 & 4.12 & -8.28 & -7.88 \\
\hline OVSF codes & 4-elements & 428 & 7.23 & -7.45 & -5.40 \\
\hline LS codes & No & 581 & 10.1 & -8.19 & -5.84 \\
\hline LS codes & 2-elements & 622 & 10.6 & -9.88 & -5.53 \\
\hline LS codes & 4-elements & 802 & 13.39 & -10.57 & -4.49 \\
\hline
\end{tabular}

Table 2: Maximum mean carried traffic and maximum number of mobile users that can be supported by the network, whilst meeting the network quality constraints of Section 4 , namely $P_{B} \leq 3 \%, P_{F T} \leq 1 \%, P_{l o w} \leq 1 \%$ and GOS $\leq 4 \%$. The carried traffic is expressed in terms of normalized Erlangs (Erlang $/ \mathrm{km}^{2} / \mathrm{MHz}$ ) using OVSF codes and LS codes in conjunction with shadow fading having a standard deviation of $3 \mathrm{~dB}$ and a frequency of $0.5 \mathrm{~Hz}$, whilst employing adaptive modulation techniques [11] for a spreading factor of $\mathrm{SF}=16$.

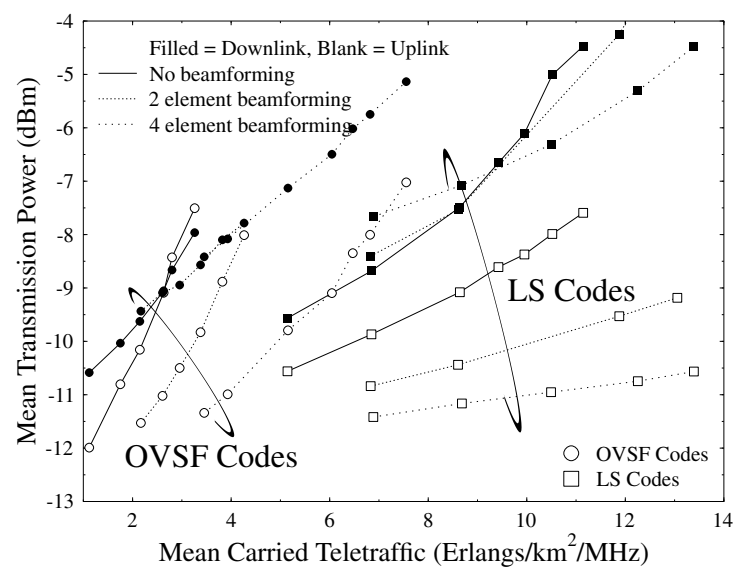

Figure 8: Mean transmission power versus number of users of the UTRA-like FDD cellular network using LS codes and OVSF codes both with as well as without beamforming in conjunction with shadowing having a frequency of $0.5 \mathrm{~Hz}$ and a standard deviation of $3 \mathrm{~dB}$ for a spreading factor of $\mathrm{SF}=16$.

to maximise the received SINR, and thus the levels of interference are attenuated efficiently. Invoking adaptive antenna arrays at the basestation reduced the mean uplink tranmission power required to meet the service quality targets of network. In OVSF codes scenarios, the basestation suffered more interference as the traffic loads increased, especially the intrainterference, which resulted uplink mean transmission power had to be increased to reach the target SINR. However, the LS codes eliminated the intra-interference superiorly, reduced the levels of interference, ultimately leading to the reduction of the mean transmission.

A summary of the maximum user capacities of the UTRAlike networks using OVSF codes and LS codes in conjunction with log-normal shadowing having a standard deviation of 3 $\mathrm{dB}$ and a frequency of $0.5 \mathrm{~Hz}$, both with and without employing beamforming is given in Table 2. The teletraffic carried and the mean mobile and base station transmission powers required are also shown in Table 2.

\section{SUMMARY AND CONCLUSIONS}

It was demonstrated that the network performance of UTRAlike system employing LS spreading codes was substantially better than that of the system using OVSF codes. Explicitly, a low dropping probability, low mobile and base station transmission power and high call quality has been maintained. In the context of the interference limited 3G CDMA system LS codes [7] might hold the promise of an increased network capacity without dramatic changes of the $3 \mathrm{G}$ standards. Our future research will focus on studying Large Area Synchronised (LAS) codes in conjunction with adaptive antenna arrays [1] and adaptive modulation techniques [4].

\section{REFERENCES}

[1] J.S. Blogh, L. Hanzo: Third-Generation Systems and Intelligent Wireless Networking - Smart Antennas and Adaptive Modulation, John Wiley and IEEE PRESS, 2002

[2] J. S. Blogh, L. Hanzo, "The Network Performance of Multi-Rate FDD-Mode UMTS", IEEE VTC'01 Fall, Atlantic City, USA, October, 2001, pp 1294-1298

[3] J. S. Blogh, L. Hanzo, "Adaptive Antenna Assisted Network Performance of FDD-Mode UMTS", IEEE VTC'01 Spring, Rhodes, Greece, May, 2001, pp 2455-2459

[4] L. Hanzo, C.H. Wong, M.S. Yee: Adaptive wireless transceivers: Turbo-Coded, Turbo-Equalised and Space-Time Coded TDMA, CDMA and OFDM systems, John Wiley and IEEE PRESS, 2002, pp 737

[5] F. Adachi, M. Sawahashi, K. Okawa, "Tree-structured Generation of Orthogonal Spreading Codes with Different Lengths for Forward Link of DS-CDMA Mobile", Electronics Letters, Vol.33, No.1, pp 27-28, 1997

[6] S. Stańczak and H. Boche and M. Haardt, “Are LAS-codes a miracle?", GLOBECOM '01, Vol. 1, pp 589-593, San Antonio, Texas, November 2001

[7] D. Li, "A high spectrum efficient multiple access code", Chinese Journal of Electronics, Vol. 8, pp 221-226, July 1999

[8] C.-C. Tseng and C. L. Liu, "Complementary Sets of Sequences",IEEE Transactions on Information Theory, Vol.18, No.5, pp 644-652, Sep. 1972

[9] R. L. Frank, "Polyphase Complementary Codes", IEEE Transactions on Information Theory, Vol.26, No.6, pp 641-647, Nov. 1980

[10] R. Sivaswamy, "Multiphase Complementary Codes", IEEE Transactions on Information Theory, Vol.24, No.5, pp 546-552, Sep. 1987

[11] L. Hanzo, L.L. Yang, E. L. Kuan and K. Yen: Single- and Multi-carrier CDMA, John Wiley and IEEE Press, to appear.

[12] S. Verdú, Multiuser Detection. Cambridge University Press, 1998.

[13] M.M.L. Cheng and J.C.I. Chuang, "Performance evaluation of distributed measurement-based dynamic channel assignment in local wireless communications", IEEE JSAC, VOL.14, May 1996 pp 698-710 